\title{
Diagnostic Development on NSTX
}

\author{
D. Johnson, R. Kaita, A. L. Roquemore, and the NSTX Team \\ Princeton Plasma Physics Laboratory \\ $\mathrm{P}, \mathrm{O}$, Box 451, Princeton, NJ 08543
}

Abstract- Diagnostics are described which are currently installed or under active development for the newly commissioned NSTX device. The low aspect ratio $(\mathrm{R} / \mathrm{a} \leq 1.3)$ and low toroidal field $(0.1-0.3 \mathrm{~T})$ used in this device dictate adaptations in many standard diagnostic techniques. Technical summaries of each diagnostic are given, and adaptations, where significant, are highlighted.

\section{INTRODUCTION}

The purpose of this paper is to describe the near term plans for diagnosing the National Spherical Torus Experiment (NSTX) plasma. It will briefly describe diagnostic systems currently installed or being actively developed. It will give particular emphasis to new techniques, or new twists on standard techniques. The spherical torus (ST) is a close cousin to the tokamak, and benefits from a long history of tokamak diagnostic development. ST specific diagnostic challenges will be highlighted, as will other cases, where measurement difficulties are eased for the ST.

As it's name implies, NSTX is a national team effort, with PPPL as the host institution. Many of the diagnostics are being developed by collaborators. A complete listing of the diagnostics being discussed is given in Table 1, with team institutions identified.

\section{NSTX DEVICE}

The NSTX is an ultralow-aspect-ratio torus $(\mathrm{R} / \mathrm{a} \leq 1.3)$ designed to produce and study high $\beta_{\mathrm{T}}$ plasmas that are noninductively sustained. ${ }^{1,2}$ Heating and current drive will be done with a 3 source neutral beam (5 MW) and a 12 strap, high-harmonic-fast-wave (HHFW) antenna $(6 \mathrm{MW})$. The goal is to produce high- $\beta_{\mathrm{T}}(25-$ $40 \%)$, low-collisionality, high-bootstrap-fraction $(\leq 70 \%)$ plasmas. In addition, coaxial-helicity-injection (CHI) will be used for noninductive plasma current initiation. Basic device capabilities are $R=0.85 \mathrm{~m}, \mathrm{a}=0.68 \mathrm{~m}, \mathrm{I}_{\mathrm{p}}=1.0 \mathrm{MA}$ $\mathrm{B}_{\mathrm{T}}=0.3 \mathrm{~T}$ and $\kappa=2.0$, with a maximum pulse length of 5 sec. Central plasma parameters in the range of $n_{e}=4$ $5 \times 10^{19} \mathrm{~m}^{-3}$ and $\mathrm{T}_{\mathrm{e}}, \mathrm{T}_{\mathrm{I}}=3-4 \mathrm{keV}$ are expected for high power heating scenarios. Wall stabilization is achieved with a close fitting set of conducting passive stabilizer plates. The device will operate in either single or double null diverted configurations. The passive plates, divertor plates and center stack (CS) are covered with carbon tiles. These structures are designed to be baked to $350^{\circ} \mathrm{C}$, and the vacuum vessel itself to $150^{\circ} \mathrm{C}$.

NSTX began as a project in late 1996, and achieved first plasma in February, 1998. Following an outage to install the passive plates, divertor plates, and protective tiles, initial research operations began in September, 1999 The Phase 1 research goals are to characterize ohmic plasmas, achieve plasma initiation with $\mathrm{CHI}$, and obtain significant plasma heating with HHFW. Installation of the neutral heating beam will occur in late 2000 .

\section{DIAGNOSTIC ACCESS}

As shown in Fig. 1, there is good diagnostic access in the midplane region, with ports very close to the plasma edge, These ports exist in 5 of 12 toroidal bays between rather narrow TF coils. Smaller ports exist in the upper and lower dome regions, although sightlines from these areas are limited, due to the $\sim 5 \mathrm{~cm}$ wide vertical cuts between passive plate sections. Fig. 2 is a plan view of the machine layout showing many diagnostic positions as well as that of the heating beam and RF antennas. Since beam spectroscopy has become one of the most powerful diagnostic tools, a midplane port was dedicated to viewing the beam with optimum geometry. In addition, special ports were designed into the domes above and below the beam trajectory, to provide vertical views of the beam.

\section{MAGNETICS}

The plasma current, shape and position is determined with inputs from a host of magnetic sensors to an equilibrium code EFIT, which also contains the geometry and currents of the NSTX coil set. Presently, there are 64 flux loops, $27 \mathrm{~B}_{\mathrm{Z}}$ coils, and 11 Rogowski coils installed.

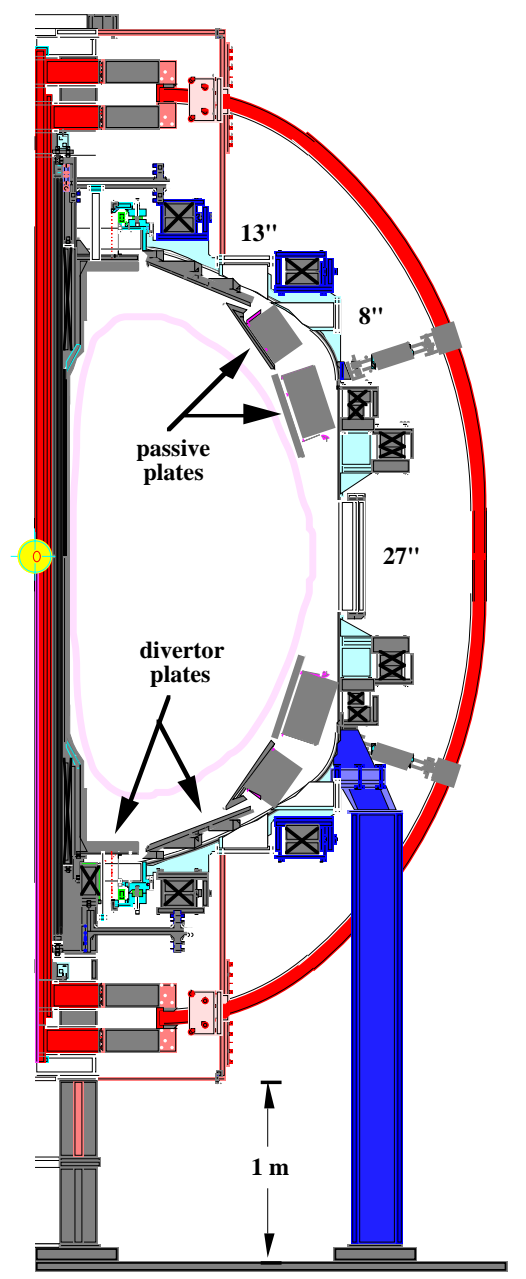

Fig. 1. Poloidal cross section of NSTX, showing internal components and diagnostic access port sizes. 
Many of these sensors are mounted on the center stack. On the air side of the CS, there are 2 plasma current Rogowskis ${ }^{3}, 4$ halo current Rogowskis, and 17 flux loops. These sensors had to fit in a radial space of $3.4 \mathrm{~mm}$ between the $\mathrm{OH}$ solenoid and the insulation between the solenoid and the inconel CS vessel wall. On the vacuum side of the CS, there are 4 halo current Rowgowskis, and $15 \mathrm{~B}_{\mathrm{Z}}$ coils arranged in one toroidal and one poloidal array. These coils are mounted in small cutouts on the backs of the $1.3 \mathrm{~cm}$ thick CFC tiles. To protect the $\mathrm{OH}$ solenoid, these tiles are thermally isolated, and during high power operation, they are radiatively cooled. It is predicted that through the course of a day of high power operation, the tile temperature will climb to $>500^{\circ} \mathrm{C}$ at some locations. The $\mathrm{B}_{\mathrm{Z}}$ coils are made with ceramic insulated wire, wound on macor mandrels, and high temperature lead wire runs through channels in the backs of the tiles. ${ }^{4}$. There are also presently 32 thermocouples mounted in the CS tiles. Coaxial helicity injection requires the centerstack to be electrically isolated (to 5 $\mathrm{kV}$ ) with large ceramic breaks between the CS and the outer vessel. Therfore, CS sensor leads must be carefully segregated, and electronics racks must be specially isolated.

The poloidal $\mathrm{B}_{\mathrm{Z}}$ coil array described above is continued outward on the passive plates, with 12 additional sensors. Space constraints are not as severe on the outer plasma facing components, so the outer $B_{z}$ coils are larger versions of the inner coils. The same materials were used, since the divertor plates and passive plates are designed to be baked to $350^{\circ} \mathrm{C}$. In conjunction with fast digitizers, the $B_{7}$ coil arrays are also useful indicators of high frequency Mirnov activity.

To provide poloidal magnetic field measurements for equilibrium reconstruction, and to derive the toroidal currents flowing in the passive plates and the outer vacuum vessel, a large array of flux loops has been installed. There are 16 loops mounted in tubes pressed into grooves on the back of the copper passive plates. Ten similar tubes are arrayed on the inside surface of the vacuum vessel and 14 loops are mounted on the outside surface. Nine additional loops are mounted on the poloidal field coils.

Many of these sensors also provide parallel input to a digital plasma control system based on a Skybolt computer with fast array input capability. ${ }^{5}$ Recently, this system has achieved closed loop control of the plasma current, radial, and vertical position.

\section{EDGE PROBES}

Fixed Langmuir probes will be used to determine divertor inner and outer strike point locations and plasma temperatures and densities in the divertor region. Presently there are 14 flush-mount Langmuir probes mounted on the center stack and inner divertor tiles. 10 additional flush mount probes are mounted in tiles in the outer divertor region.

A pneumatically driven, fast scanning Langmuir probe array will be installed to study the plasma edge, in a region extending a few centimeters inboard and outboard of the outer separatrix. This probe is based on designs used previously on TEXTOR ${ }^{6}$, PBX-M, and DIII-D to study edge electron temperature, plasma density, and floating potential profiles, as well as fluctuations in these quantities. The drive has a maximum insertion speed of 3 $\mathrm{m} / \mathrm{s}$. Measurements with $\sim 2 \mathrm{~mm}$ spatial resolution and 200 $\mathrm{kHz}$ bandwidth are possible. This diagnostic will be very useful in characterizing edge transport and turbulence in all regimes, and for achieving conditions for optimal RF coupling in HHFW heating and current drive experiments.

Two CS tiles have been modified and coupons have been placed at various positions. These will undergo surface analysis after exposure to study erosion and deposition. The CS tiles were implanted with silicon depth markers. Half of the area of $2.5 \mathrm{~cm}$ square, SS coupons were coated with $\sim 300 \mathrm{~nm}$ of carbon. 16 such coupons along with 2 silicon coupons are arrayed on the outer vessel and in gaps between the passive plates.

\section{VISIBLE AND IR IMAGING CAMERAS}

Because of the small size of the center column, and the proximity of the midplane ports to the plasma, with a sufficiently wide angle view from a midplane window, a large fraction $(>90 \%)$ of the plasma can be seen, in contrast to similar views on standard tokamaks. An intensified, fast framing CCD camera equipped with such a view has been extremely useful during the commissioning of the plasma control system. This system consists of a fisheye $2.8 \mathrm{~mm}$ FL lens imaging onto a 10' coherent fiber bundle needed to bring the image out of the high magnetic field area. The bundle output is reimaged through an interference filter onto a Kodak EKTAPRO EM1012 camera, capable of $1000 \mathrm{fps}$ and storage for $\sim 1600$ frames, and exposure times as short as 10us. The fast camera is complemented by a high resolution standard frame rate CCD camera with a similar fisheye view mounted directly behind a low profile midplane window. These cameras provide valuable operational feedback on plasma shape and position, and the character of the plasma edge and wall interaction.

The fast camera described above will also be used to study edge turbulence, with a narrower field of view. ${ }^{7}$ An orifice has been located at a position displaced appropriately from the camera to provide a gas puff which can be viewed along an edge field line during nominal plasma conditions. A puff of $\mathrm{D}$ or $\mathrm{He}$ gas will be used to illuminate 2-D edge density turbulence, for example, during $\mathrm{L}$ to $\mathrm{H}$ confinement transitions.

During high power operation, IR cameras will be used to view power deposition on the CS and in the divertor regions. This is particularly important for other components near the midplane, such as the RF antennas and the neutral beam armor, since calculations indicate that significant heating will occur in these areas due to prompt loss of fast ions (see 'fast ion diagnostics' below). Current IR camera capability includes two Inframetrics 525 cameras with $\mathrm{HgCdTe}$ detectors, operating from 8-12 $\mu$. These cameras have a temperature range from $-20^{\circ} \mathrm{C}$ to $1500^{\circ} \mathrm{C}$ with a $30 \mathrm{~Hz}$ frame rate.

Passive spectroscopy provides the tools, for example, to monitor wall conditioning trends, to understand the causes of sudden impurity influxes, to perform quantitative particle balances, and to assess $\mathrm{Z}_{\text {eff }}$ and $\mathrm{P}_{\mathrm{rad}}$ profiles.

Two survey spectrometers previously used on TFTR have been relocated to NSTX. VIPS is a $0.64 \mathrm{~m}$ CzernyTurner visible survey instrument with spectral coverage from $185-750 \mathrm{~nm}$, configured either with a midplane view across the machine, or with a view of one of several optical fibers with relocatable viewing telescopes. SPRED $^{8}$ is a VUV survey spectrometer tunable over a range $10-110 \mathrm{~nm}$ with midplane view. Both of these instruments use 1024 element reticon array detectors with full frame time of $17 \mathrm{~ms}$.

A 16 channel bolometer array is installed with a midplane tangential fan ranging from the edge of the CS to the outer plasma edge. The instrument is similar to several at the C-Mod tokamak, ${ }^{9}$ consisting of pinhole $(\sim 0.2 \mathrm{~mm})$ optics illuminating an International Radiation Detectors (IRD) 16EL0 AXUV silicon photodiode array, with data acquired at $5 \mathrm{kHz}$. 
Several fiberscopes are mounted on the machine for use with interference filters to monitor $\mathrm{D}_{\alpha}, \mathrm{CII}, \mathrm{CIII}$, and HeII. One of these is used as a visible bremsstrahlung monitor at $523 \mathrm{~nm}$, and views with a narrow beam across the midplane into a long pumping duct, which serves as an effective viewing dump.

\section{ACTIVE SPECTROSCOPY}

\section{Thomson Scattering}

Detailed $T_{e}$ and $n_{e}$ profile measurements will be essential for many NSTX experiments. It was recognized that conventional ECE techniques would not be applicable at the low toroidal fields of NSTX (see further discussion below in microwave diagnostics section), and so Thomson scattering was chosen to provide time resolved $T_{e}(R)$ measurements. The need for multipulse capability led to the choice of a Nd:YAG based system, similar to many in use on tokamaks.

A system is currently being installed which uses a backscattering geometry, which gives full radial coverage on the horizontal midplane, with high sensitivity and high spatial resolution at the outer edge. ${ }^{10} \mathrm{~A}$ feature of this geometry is that the viewing system does not see the center stack tiles, or the top or bottom divertor tiles. During high power, long pulse operation, these surfaces will get hot enough to blind systems sensitive at $1 \mu \mathrm{m}$. The $30 \mathrm{~Hz}, 1.6$ joule/pulse

\begin{tabular}{|c|c|c|c|}
\hline Type & Diagnostic & Phase & Lead \\
\hline \multirow[t]{6}{*}{ Equilibrium } & Flux loops & 1 & PPPL \\
\hline & Rogowski coils & 1 & PPPL \\
\hline & Bz coils & 1 & PPPL \\
\hline & Slow plasma TV & 1 & PPPL \\
\hline & Fast plasma TV & 1 & LANL \\
\hline & Locked mode coils & 1 & PPPL \\
\hline \multirow[t]{3}{*}{ Basic } & $2 \mathrm{~mm}$ interferom. & 1 & PPPL \\
\hline & X-ray PHA & 1 & PPPL \\
\hline & X-ray crystal spect. & 1 & PPPL \\
\hline \multirow[t]{5}{*}{ Impurity } & VIPS visible spect. & 1 & PPPL \\
\hline & SPRED VUV spect. & 1 & PPPL \\
\hline & Single channel VB & 1 & PPPL \\
\hline & Filtered fiberscopes & 1 & ORNL \\
\hline & VB array & 2 & PPPL \\
\hline \multirow[t]{8}{*}{ Profile } & EBW radiometer & 1 & PPPL \\
\hline & Thomson scattering & 2 & PPPL \\
\hline & Reflectometer & 2 & UCLA \\
\hline & FIR tang interf/polar & 2 & UCD \\
\hline & Toroidal CHERS & 2 & PPPL \\
\hline & MSE (CIF) & 2 & FP\&T \\
\hline & Poloidal CHERS & 3 & PPPL \\
\hline & MSE (LIF) & 3 & FP\&T \\
\hline \multirow[t]{3}{*}{ MHD } & USXR arrays & 1 & JHU \\
\hline & SXR arrays & 2 & PPPL \\
\hline & Fast tang. XR camera & 2 & PSI \\
\hline \multirow[t]{7}{*}{ Edge/Divertor } & IR Cameras & 1 & PPPL \\
\hline & Fast scanning probe & 1 & UCSD \\
\hline & SOL reflectometer & 1 & ORNL \\
\hline & Edge reflectometer & 1 & UCLA \\
\hline & Langmuir probes & 1 & PPPL \\
\hline & Edge coupons & 1 & SNL \\
\hline & Fast Edge Imaging & 1 & LANL \\
\hline \multirow[t]{2}{*}{ Fast Ions } & Fast ion loss probe & 2 & PPPL \\
\hline & Scanning NPA & 2 & PPPL \\
\hline
\end{tabular}

Table 1. NSTX Diagnostics currently installed or under development, the phasing of this development, and the lead institutions.
Spectra Physics laser is directed through the machine on the horizontal midplane at $\mathrm{R}_{\mathrm{TAN}}=28 \mathrm{~cm}$. It exits the machine along a long pumping duct and out a window to an absorbing dump. The viewing system consists of a 16 $\mathrm{cm}$ diameter window and circular aperture with a $68 \mathrm{~cm}$ radius spherical mirror, which images the laser beam onto a curved array of 36 fiber optic bundles. The bundles transport the imaged light to filter polychromators housed in an accessible, shielded room near the laser. Spatial resolution will be $\sim 1.0 \mathrm{~cm}$ at the plasma outer edge, 3-4 $\mathrm{cm}$ at the magnetic axis, and $8-10 \mathrm{~cm}$ at the inner edge.

Initially, there will be a single $30 \mathrm{~Hz}$ laser and 10 polychromators (spatial positions). As resources permit, the system will be upgraded to utilize three lasers and the full 36 spatial channels.

Part of the motivation to install the heating neutral beam on NSTX was to allow beam spectroscopy, initially for charge exchange recombination spectroscopy for measuring impurity ion temperature and flow velocity, and for motional stark effect polarimetry to measure $\mathrm{B}_{\theta}$ and $\mathrm{E}_{\mathrm{r}}$. Another powerful fluctuation measurement technique, beam emission spectroscopy, (BES) was also considered. However, the BES technique relies on being able to view the beam nearly along field lines. Due to the very large magnetic field pitch on the ST $\left(\sim 50^{\circ}\right.$ at outer midplane separatrix for 1.0 MA), and its large variation in space and time, this technique is problematic with a single view on NSTX.

Charge exchange recombination spectroscopy (CHERS)

A tangentially viewing CHERS system is currently being designed for installation at the time of initial NBI operation, to provide profiles of $T_{i}$ and $v_{\phi}$. This system will share the same viewing optics with the motional-starkeffect system, described below. The geometry is chosen to optimize the spatial resolution at the plasma edge, where it will be $\sim 0.5 \mathrm{~cm}$, increasing to $\sim 3.0 \mathrm{~cm}$ at the plasma core. The system will have $\sim 75$ channels with a concentration of channels in the outer $10 \mathrm{~cm}$, and will reuse the high throughput Kaiser spectrometers and Princeton Instruments Pentamax CCD cameras used formerly on the TFTR poloidal rotation diagnostic. ${ }^{11}$

Design work is beginning on a vertically viewing CHERS system with matched top and bottom views to provide $\mathrm{v}_{\theta}$ measurements. This system will be very similar to that described in ref. 9. Using the data from both of the CHERS systems along with the radial force balance equation, one can calculate the radial electric field, $E_{r}(R)$, an important parameter affecting plasma confinement in tokamaks through ExB shear flow suppression of turbulence. This phenomenon is expected to be even stronger in lower aspect ratio ST devices. (see ref. 1)

\section{Motional Stark Effect Polarimetry}

Since noninductive current initiation and sustainment are top level NSTX research goals, measurements of the current profile $\mathrm{J}(\mathrm{R})$ are essential to many planned experiments. On tokamaks with neutral beams, the standard technique for this task is motionalstark-effect polarimetry (MSE), ${ }^{12,13}$ which recently has also been critical in measurements of $E_{r}(R)$.

The MSE technique relies upon the spectral splitting of the Balmer- $\alpha$ line into othogonally polarized components. This Stark splitting is a result of the strong electric field in the rest frame of the injected deuterium atoms, induced by their motion through the confining magnetic field $\left(\mathbf{E}=\mathbf{v}_{\mathrm{B}} \mathrm{x} \mathbf{B}\right)$. Detecting the polarization angle of one of these components provides a measure of the local magnetic field direction. To date, MSE measurements using conventional techniques are limited 
to $\mathrm{B} \geq 0.8 \mathrm{~T}$, due to the difficulty of spectrally isolating the polarization components.

On NSTX several modifications are planned to adapt the technique to lower field. ${ }^{14}$ First, the Doppler broadening of the individual components will be minimized, by reducing the spread in angles between the beam and the viewing aperture. This will be done by narrowing the viewing aperture in the horizontal dimension, and to compensate, vertically elongating the aperture. Second, a narrower bandwidth birefringent filter is being developed, based on a Lyot design currently utilized in solar spectroscopy and dye laser tuning. The goal of this development is an effective passband width of $0.075 \mathrm{~nm}$, compared to $0.7 \mathrm{~nm}$ width of standard interference filters. Modeling the performance of a system, with the new aperture shape and a $0.075 \mathrm{~nm}$ filter passband, one obtains polarization fractions of $\mathrm{P}_{\mathrm{f}}=0.2-0.4$ for the central $\sigma_{0}$ component. Typical MSE systems operate with a $\mathrm{P}_{\mathrm{f}} \sim 0.8$, and, to compensate, the optical throughput on the NSTX system will be much larger than on previous systems. The optics is designed to image the beam onto 20 fiber optic bundles, providing spatial resolution of $2.5 \mathrm{~cm}$ at the edge and $3.0 \mathrm{~cm}$ in the core.

The emission used in MSE systems results from collisionally-induced-fluorescence (CIF) arising from collisions between injected D atoms and the background plasma. Using a tunable laser to induce this emission can have significant advantages. (see ref. 12) On a parallel but somewhat delayed development path to the MSE work described above, development is underway on laserinduced-fluorescence (LIF) MSE. The plan is to inject a hydrogen diagnostic neutral beam with a coaxial tunable laser resonant with the Doppler shifted $\mathrm{H}_{\alpha}$ transition. With a narrow spectral width, polarization fractions near unity are predicted even at the low fields of NSTX. Lab tests are underway with a beam designed to operate at 40 $\mathrm{keV}$ and $30 \mathrm{~mA}$, with a $1.2 \mathrm{~cm}$ diameter.

The intrisic electric field, $E_{r}$, can also affect the direction of the polarization, and this fact has been recently exploited (see refs. 9, 10) to measure both the magnetic field angle and the radial electric field. This requires using multiple MSE views or viewing different beam components. If the CIF and LIF MSE systems described above are successful on NSTX, determination of both parameters will be possible.

It is interesting to note that the design of the collection optics for all of the active spectroscopy systems described above were severely constrained by the presence of the TF coils. In these cases and several others, a more optimum port configuration would have placed the ports directly inboard of the TF coils, more easily permitting tangential views.

\section{X-RAY DIAGNOSTICS}

$\mathrm{X}$-ray survey spectra in the energy range $1-50 \mathrm{keV}$ are measured using a $\mathrm{x}$-ray pulse height analyzer (PHA) previously used on TFTR. ${ }^{15}$ Six different $\mathrm{Si}(\mathrm{Li})$ detectors measure different ranges of photon energy with a resolution of $250 \mathrm{ev}$. The horizontal sightline is at the midplane along the axis of the pumping duct. The electron temperature is derived from the slope of the $\mathrm{x}$-ray continuum radiation, and $\mathrm{Z}_{\text {eff }}$ due to metals can be estimated from $x$-ray line emission.

At each of two toroidal locations, $90^{\circ}$ apart, a set of three arrays of discrete, large area IRD AXUV silicon photodiodes will be installed, to study MHD activity and plasma turbulence. In each set, two of the arrays are mounted on the large midplane ports viewing the upper and lower halves of the plasma, and a third is mounted on a top flange, viewing the plasma from above. The array enclosures are mounted behind gate valves, readily permitting modification of the detector configurations.
Each enclosure will house 16 large area AXUV diodes, arrayed on an arc around a viewing slot. A moveable slide behind the slot houses several thin foil filters.

The first set of arrays, just installed, will be configurable with multilayer mirrors, for high spectral resolution ( $\leq 2 \AA)$ imaging. ${ }^{16}$ These will be particularly useful for rejection of the bright core emission, when trying to study MHD and turbulence in the periphery. One could, for example, image the C IV Ly $\alpha$ emission at $33.7 \AA$ with a $\mathrm{Ti}$ foil filter- $\mathrm{Ti} / \mathrm{Cr}$ mirror-diode arrangement, with an estimated back ground rejection factor of $\sim 100$.

A second set of arrays, without multilayer mirrors, but with an identical viewing geometry, will be used to study core MHD, and will be installed before the NBI run. When configured with the same detector arrangement, the two array sets would be useful in mode identification of core MHD. Equipped with different filters, they could also provide a fast estimate of $T_{e}(R)$ from the two color technique.

An x-ray crystal spectrometer is being installed to measure core ion temperature, using non-perturbing puffs of Ar gas. ${ }^{17}$ The design uses a Johann geometry and a horizontal diffraction plane. It will accommodate either a cylindrically-bent or a spherically-bent crystal. With the latter, and a 2-D imaging detector, many vertically displaced sightlines can be simultaneously analyzed. The dielectric satellite spectrum of Helium-like ArXVII will be used to derive values of the Ar ion temperature and the electron temperature. This diagnostic will be particularly useful in studies where $T_{\mathrm{I}}$ information from CHERS are not available, such as ohmic and RF-only experiments.

Design work has begun on a fast tangential 2-D xray camera system, similar to that recently developed for TEXTOR. ${ }^{18} \mathrm{X}$-rays would be imaged by a pinhole onto a CsI scintillator faceplate, which is coupled to a $100 \mathrm{~mm}$ diameter Hamamatsu electrostatic image intensifier. A fiber optic reducer couples the P47 phosphor intensifier output to a fast frame rate $\mathrm{CCD}$. The PSI $\mathrm{CCD}^{19}$ is designed with $64 \times 64$ superpixels, each $200 \mu$ square, with $40 \%$ active area. Each superpixel has a storage register to store 300 frames. The effective frame rate will be up to $\sim 500 \mathrm{kHz}$. This camera will be used in a tangential viewing geometry, which should allow high m-modes (m 20) to be resolved with good accuracy. It is predicted that MHD events which change the x-ray emissivity of order $1 \%$ over a few cm could be resolved with $100 \mathrm{kHz}$ time resolution with this system.

\section{MICROWAVE and FIR DIAGNOSTICS}

Line density measurements are made with a 170 $\mathrm{GHz}$ interferometer system adapted from part of the TFTR correlation reflectometer ${ }^{20}$. The beam is launched radially through a midplane window, reflects off the carbon CS tiles, and is received through the same window.

A millimeter wave reflectometer system is being installed with a goal to measure density profiles in swept FM mode, and fluctuations in fixed frequency mode. An array of 4 pairs of transmit/receive horns has been installed on a midplane port covering the range $12-50$ $\mathrm{GHz}$, accessing densities in the range $2 \times 10^{12}-3 \times 10^{13} \mathrm{~cm}^{-3}$. The horns are capable of launching arbitrary plane polarized waves. Because of the large field pitch and field shear in the ST, particularly for high $\beta$ operation, mode mixing could have a significant impact on data interpretation. Experimental data with both $\mathrm{X}$ - and $\mathrm{O}$ mode launch will be compared with numerical simulations to improve understanding in this area. The reflectometer will be a valuable tool for studying phenomena involving fast changes in the edge density profile, such as L-H transitions and ELMS as well as edge turbulence.

A lower frequency reflectometer for studying density profiles in the scrapeoff layer has horns integrated into the 
HHFW antennas. This system has a frequency range 3-36 $\mathrm{GHz}$, sweepable in 100-850 $\mu$ s. The fast sweep time will aid in avoiding problems with large density fluctuations in this region. Once again, cylindrical waveguides are used near the machine, and the polarization can be adjusted to match to the edge pitch angle. This system will be used to optimize RF antenna coupling to the plasma.

Electron cyclotron emission (ECE) radiometry has been a powerful diagnostic tool in tokamaks, providing temporally and spatially resolved measurements of electron temperature. On NSTX, the toroidal field is low enough that $\Omega_{\mathrm{ce}}<\omega_{\mathrm{pe}}$, and electromagnetic radiation is not generated for the first several cyclotron harmonics.
Radiation at higher harmonics will propagate, however, it does not satisfy the blackbody condition necessary to make it a good monitor of electron temperature. A promising new development for diagnosing electron temperature in low field plasma confinement devices is electron Bernstein wave (EBW) radiometry. ${ }^{21}$ For NSTX conditions, EBW waves propagate in the plasma and meet the blackbody criterion. This concept has recently been tested on the $\mathrm{CDX}-\mathrm{U}$ device operating at $0.2 \mathrm{~T}$, where a manually tunable device operating between 8-10 GHz, has obtained initial $\mathrm{T}_{\mathrm{e}}$ profile data, with an averaging time of $0.5 \mathrm{~ms}$. An antenna is mounted outside a quartz window, viewing second harmonic EBW emission, mode converted to

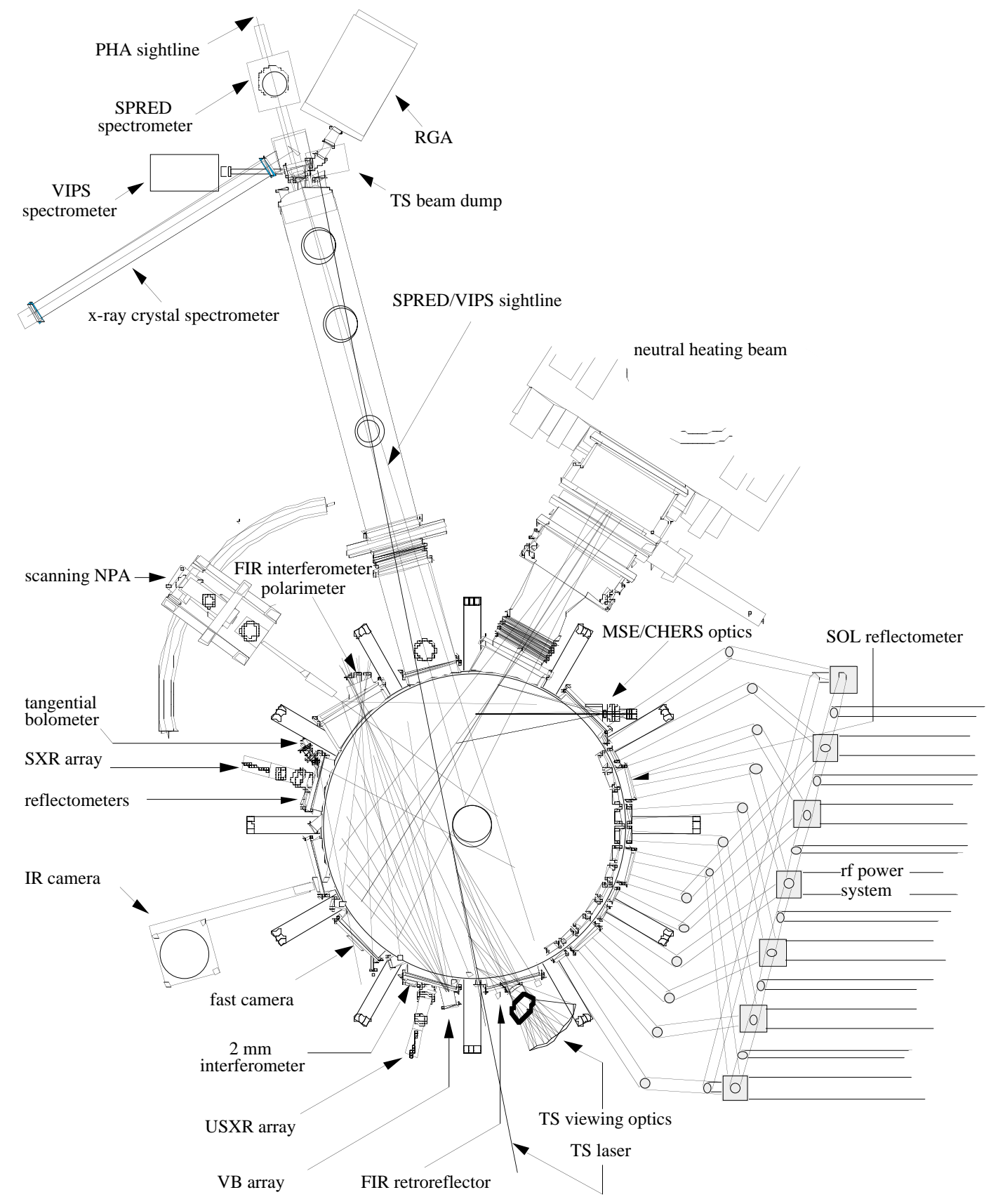

Fig. 2 Plan view of diagnostic layout and sightlines on NSTX 
$\mathrm{X}$-mode electromagnetic radiation at the upper hybrid layer near the plasma edge. This system will soon be installed to make similar measurements on NSTX.

Design is underway for a 7 channel, tangential, FIR laser interferometer/polarimeter for NSTX. ${ }^{22}$ Fully implemented, the diagnostic will utilize two probing lasers with opposite circular polarizations, and a third reference laser, all operating at $119 \mu$. Phase shiftsbetween the two probing lasers are due to the Faraday effect, and provide information on the toroidal field. The average phase shift relative to the reference beam is a measure of the refractive index, providing information on the electron density. With spatial inversion, $\mathrm{n}_{\mathrm{e}}(\mathrm{R}, \mathrm{t})$ and $\mathrm{B}_{\mathrm{T}}(\mathrm{R}, \mathrm{t})$ profiles can be obtained with time resolution up to 100 $\mathrm{kHz}$. Core sightlines will typically see 10-20 fringes of density signal and $5-15^{\circ}$ of Faraday rotation. The system should have excellent $\mathrm{S} / \mathrm{N}$, since minimum detectable signals are predicted to be .03 fringes and .03 degrees. Because of the low toroidal field on NSTX, the deviation in the vacuum toroidal field in the core due to plasma diamagnetism is predicted to be $\sim 100$ gauss for high $\beta$ discharges. This deviation represents a few degrees of Faraday rotation, and should be readily observable. This measurement will provide valuable constraints on the equilibrium reconstruction of NSTX plasmas. Initially, two sightlines will be used with retroreflectors located outside the vacuum, on the stable Thomson scattering viewing platform.

\section{FAST ION DIAGNOSTICS}

The low value of the magnetic field in NSTX leads to the existence of a rich variety of energetic ion phenomena, such as high energetic particle beta, large population of super Alfvénic ions, large Larmor radii and orbit widths, large population of trapped ions, anisotropy induced by prompt loss of edge or counter-going beam and/or RF-driven ions and possiblity of significant loss of energetic particles due to MHD interactions. Measurements of the ion distribution in energy, space and pitch angle using a charge exchange neutral particle analyzer (NPA) can provide essential information to support evaluation of these effects.

The passive charge exchange diagnostic for NSTX uses an E\|B-type NPA originally designed for TFTR ${ }^{23}$. The $E \| B$ spectrometer has an energy range of $0.5 \leq$ $\mathrm{A}(\mathrm{amu}) \mathrm{E}(\mathrm{keV}) \leq 600$ and for the NSTX application provides mass-resolved energy spectra of $\mathrm{H}^{+}$and $\mathrm{D}^{+}$ion species simultaneously during a single discharge. The detector consists of a large-area microchannel plate which is provided with two rectangular, semi-continuous active area strips, one coinciding with each of the mass rows for detection of $\mathrm{H}^{+}$and $\mathrm{D}^{+}$. Each mass row has 39 energy channels and has an energy dynamic range of $\mathrm{E}_{\max } / \mathrm{E}_{\min }=$ 30. The energy resolution is $3 \% \leq \mathrm{E} / \mathrm{E} \leq 7 \%$. Time resolution, is expected to be $\sim 5 \mathrm{~ms}$. To facilitate the measurements noted above, the design of the support structure for the NPA provides horizontal and vertical scanning capability to map the ion energy distribution in space and pitch angle.

Predictions indicate that $10-30 \%$ of the neutral beam heating power will be lost in moderate-to-high $\beta, 1.0$ MA NSTX plasmas, due to the large orbit width and Larmor radius of these ions. Modeling of prompt $80 \mathrm{keV}$ beam ion loss rates indicate that peak fluxes in the range of $\sim 10 \mathrm{MW} / \mathrm{m}^{2}\left(\sim 10 \mathrm{~mA} / \mathrm{cm}^{2}\right)$ occurs on surfaces near the outer plasma edge, such as the neutral beam armor and the RF antennas. IR camera views of these regions will map out resulting surface heating. A fast ion loss probe is being designed to measure the time behavior of the fast ion edge flux. Fast ion currents to thin metal films in an alternating metal/insulator film collector will be measured to provide energy resolution. For example, a structure consisting of vacuum-deposited $\mathrm{Al}$ and $\mathrm{SiO}_{2}$ layers, each layer several hundred $\mathrm{A}$ thick is being considered. The collector will be mounted behind a slit in a probe tip with a drive that is radially scannable at the midplane between shots.

\section{CONCLUSION}

A national team is assembling a broad array of diagnostics for the newly commissioned NSTX device, which have been summarized. Most techniques familiar to tokamak research are also adaptable to spherical tori. However, the low toroidal field and the low aspect ratio of the ST dictate changes to some standard techniques and development of some new techniques, examples of which have been highlighted. As the NSTX research program continues to mature, ongoing and future diagnostic development will be a key component of the program, leading to many new physics insights.

\section{ACKNOWLEDGEMENTS}

The authors wish to acknowledge the support of the NSTX Program Director, M. Peng, the NSTX Project Director, M. Ono, and the NSTX Experimental Operations Head, J. Hosea. This work funded by U.S. DoE contract DE-AC02-76-CH0-3073.

\section{REFERENCES}

${ }^{1}$ S. M. Kaye, et al., Fusion Technology, 36, 1 (1999)

${ }^{2} \mathrm{M}$. Ono, et al., paper OC1-3, this conference

${ }^{3}$ B. McCormack, et al., paper PA5-9, this conference

${ }^{4}$ H. Kugel, et al., paper PA5-8, this conference

${ }^{5}$ C. Neumeyer, et al., paper PA5-3, this conference

${ }^{6}$ J. A. Boedo, et al., Rev. Sci. Instrum., 70, 2997 (1999)

${ }^{7} \mathrm{~S}$. Zweben, private communication.

${ }^{8}$ B. C. Stratton, et al., Rev. Sci. Instrum., 57, 2053 (1986)

${ }^{9}$ R. L. Boivin, et al., Rev. Sci. Instrum., 70, 260 (1999)

${ }^{10}$ D. Johnson, et al., Rev. Sci. Instrum., 70, 776 (1999)

${ }^{11}$ R. E. Bell, et al., Rev. Sci. Instrum., 70, 821 (1999)

${ }^{12}$ F. M. Levinton, Rev. Sci. Instrum., 70, 810 (1999)

${ }^{13}$ B. W. Rice, et al., Rev. Sci. Instrum., 70, 815 (1999)

${ }^{14} \mathrm{~F}$. M. Levinton, private communication.

${ }^{15}$ K. W. Hill, et al., Rev. Sci. Instrum., 56, 840, (1985)

${ }^{16}$ D. Stutman, et al., Rev. Sci. Instrum., 70, 572 (1999)

${ }^{17}$ M. Bitter, et al., Rev. Sci. Instrum., 70, 292 (1999)

${ }^{18}$ S. VonGoeler, et al., Rev. Sci. Instrum., 70, 599 (1999)

${ }^{19}$ Under development by Princeton Scientific

Instruments, 7 Deer Park Drive, Monmouth Junction, NJ 08852, under DOE SBIR contract.

${ }^{20}$ E. Mazzucato, et al., Rev. Sci. Instrum., 63, 4657 (1992)

${ }^{21}$ P. Efthimion, et al., Rev. Sci. Instrum., 70, 1019 (1999)

${ }^{22}$ H. Park, et al., Rev. Sci. Instrum., 70, 710 (1999)

${ }^{23}$ S. S. Medley, et al., Rev. Sci. Instrum. 69, 2651 (1998) 